mol. potassium $/ \mathrm{kgm}$; during stimulation at 200 impulses/sec. there was an entry of $3.5 \times 10^{-12} \mathrm{~mol}$. sodium $/ \mathrm{cm} .{ }^{2} / \mathrm{impulse}$ and a loss of $3.0 \times 10^{-12} \mathrm{~mol}$. potassium/cm. ${ }^{2} /$ impulse. These results provide further support for the hypothesis put forward by Hodgkin, Huxley and $\mathrm{Katz}^{6,7}$ that during the rising phase of the nerve action potential there is a considerable increase in the permeability of the membrane to sodium.

We are indebted to the director and staff of the Marine Biological Laboratory, Plymouth, where the axons were collected, for their hospitality, and to the Atomic Energy Research Establishment, Harwell, for irradiating our samples. The expenses of the work were met by a grant from the Rockefeller Foundation.

R. D. Keynes

P. R. LEwis

Physiological Laboratory,

University of Cambridge.

Feb. 9.

1 Keynes, R. D., J. Physiol., 109, $13 P$ (1949).

'Keynes, R. D., C.R. Colloque d'Electrophysiologie, Paris, April 1949 (in the press).

3 Tobias, C. A., and Dunn, R. W., Science, 109, 109 (1949).

- Brown, H., and Goldberg, E., Science, 109, 347 (1949).

s Glick, D., "Techniques of Histo- and Cytochemistry" (Interscience Pub., New York, 1949).

- Hodgkin, A. L., and Katz, B., J. Physiol., 108, 37 (1949).

' Hodgkin, A. L., Huxley, A. F., and Katz, B., C.R. Colloque d'Electrophysiologie, Paris, April 1949 (in the press).

\section{Determination of Neutron Densities by the Permanganate Method}

Densities of slow neutrons in extended media may be measured by graphical ${ }^{1}$ or physical $^{2}$ integration of the $\beta$-activity induced by neutron capture in a detecting element distributed in the medium. The combination of Szilard-Chalmers concentration with physical integration greatly increases. the available intensity ${ }^{3}$. Manganese, as permanganate ion, is an eminently suitable detector ${ }^{a, 5}$. The bulk of the induced activity is found in the manganese dioxide fraction, which is isolated by simple filtration. This activity is a measure of the average neutron density.

However, a minor, but variable, part of the activity remains with the permanganate fraction, that is, in solution ('retention' 6 ). We have investigated the mechanism of the Szilard-Chalmers effect in the permanganate ion by varying the conditions of irradiation?. We wish to report here the part of our conclusions which may be of use to those desiring to apply the method. As a result of our investigations into the factors which determine retention, it seems that its value may be kept sufficiently constant for measurements of neutron densities to be reproducible within \pm 1 per cent.

Separation of the radioactive manganese dioxide by filtration through fine paper (Schleicher-Schüll Blauband) or fine glass sinter filters (Jena $G 4$ ) is complete. The efficiency of this separation is not affected by the amount of manganese dioxide carrier added after irradiation, or by the amount of suspended manganese dioxide during irradiation. No appreciable part of the activity is held back by the glass walls of containers if they are washed with hydrochloric acid. Of course, it is well known that no exchange of activity between manganese dioxide and the permanganate ion occurs at room temperature ${ }^{8}$.
Retention is independent of $p H$ value between 2 and 7 (measured with glass electrode), but increases on either side of this region. Commercial potassium permanganate always contains free alkali. Phosphate buffer should be used; but at buffer concentrations greater than $10^{-3} \mathrm{M}$, part of the active manganese dioxide will pass through the filter as a colloid ${ }^{\circ}$. The retention decreases with decreasing concentration of permanganate ion $(1.5 \mathrm{M}, 17$ per cent ; $0.25 \mathrm{M}, 12$ per cent ; $0.025 M, 6$ per cent ; $0.0025 M, 4$ per cent). These values were obtained at room temperature mostly with neutral solutions of potassium permanganate; the first value was obtained with sodium or calcium permanganate, on account of its greater solubility. Retention increases steeply with temperature. Between $10^{\circ}$ and $30^{\circ} \mathrm{C}$. it rises from 10 to 16 per cent at concentration $0.22 \mathrm{M}$, and from 5 to 6.5 per cent at $0.03 M$. At $0.22 M$ and $96^{\circ} \mathrm{C}$. retention reaches the large value of 75 per cent.

The practical advice is, then : keep the solution (defined concentration) close to $p \mathrm{H} \mathrm{5}$, use phosphate buffer $10^{-4} M$, keep the temperature reasonably constant, filter through fine paper or glass filter, and recover manganese dioxide adsorbed by the walls with hydrochloric acid.

We wish to thank Prof. K. Przibram for his interest in our work.

J. ERBER

W. RIEDER

E. BrodA

II. Physikalisches Institut der Universität, Wien. Dec. 14

Amaldi, Hafstad and Tuve, Phys. Rev., 51, 896 (1937)

Booth and Hurst, Proc. Roy. Soc., A, 161, 248 (1937). Fermi, Anderson and Szilard, Phys. Rev., 56, 284 (1939).

${ }^{3}$ Halban, Kowarski and Magat, C.R. Acad. Sci., Paris, 208, 572 (1939).

Rotblat (unpublished, 1943).

Broda, J. Chim. Phys., 45, 193 (1949).

Libby, J. Amer. Chem. Soc., 62, 1930 (1940).

Broda, Erber and Rieder, Monatsh. Chem. (in the press). Rieder, thesis, Vienna (1949). Monatsh. Chem. (in the press).

${ }^{8}$ Polissar, J. Amer. Chem. Soc., 58, 1372 (1936).

- Broda and Erber, Monatsh, Chem., 81, 53 (1950).

\section{Measurement of Appearance Potentials of Ions Produced by Electron Impact, using a Mass Spectrometer}

THE mass spectrometer is often used for the measurement of the appearance potentials of ions produced by electron bombardment of gaseous molecules. For this purpose, two gases are admitted to the instrument simultaneously; one is the gas under investigation, while the other is a 'standard gas' (usually argon or krypton), the ionization potential of which is accurately known from spectroscopic data. Measurements are made of the ion beam intensities as a function of electron energy, both for the ion the appearance potential of which is required and for that for which it is known. These measurements are made over a range of a few volts above the potentials at which the beams are first detected. Several rather arbitrary methods have been used for deducing the difference between the two potentials ${ }^{1}$.

The purpose of this communication is to describe a new method of calculation which has been adopted in these Laboratories in connexion with work on dissociation processes in certain gases and the measurement of bond energies, using a Nier-type mass-spectrometer. We find that in many cases the curve relating ion-beam intensity to electron voltage 